\title{
TEM study of boron phosphide: Discovery of rhombohedral BP
}

\author{
B.A. Kulnitskiy, ${ }^{a, b}$ V.D. Blank, ${ }^{a, b}$ T.A. Gordeeva, ${ }^{a, b}$ V.A. Mukhanov, ${ }^{c}$ and V.L. Solozhenko ${ }^{d,}$ \\ ${ }^{a}$ Technological Institute for Superhard and Novel Carbon Materials, 108840 Troitsk, Russia \\ ${ }^{b}$ Moscow Institute of Physics and Technology, 141700 Dolgoprudny, Russia \\ ${ }^{c}$ Mineral Ltd., 601650 Alexandrov, Russia \\ ${ }^{d}$ LSPM-CNRS, Université Sorbonne Paris Nord, 93430 Villetaneuse, France
}

\begin{abstract}
Microstructure of sphalerite (3C) boron phosphide, BP, produced by self-propagated hightemperature synthesis has been studied by high-resolution transmission electron microscopy. Along with numerous twins on the $\{111\}_{3 \mathrm{C}}$ plane, layers of wurtzite $(2 \mathrm{H})$ polymorphic modification and previously unknown for BP rhombohedral (3R) structure were found which indicates trimorphism of BP.
\end{abstract}

Keywords: boron phosphide, transmission electron microscopy, polymorphism, twinning.

\section{Introduction}

Boron phosphide $\mathrm{BP}$ is a hard (Vickers hardness $H_{\mathrm{V}} \sim 30 \mathrm{GPa}$ [1]) refractory (melting temperature at ambient pressure is $2840 \mathrm{~K}$ [2]) and low-compressible (300-K bulk modulus is $174 \mathrm{GPa}$ [3]) wide bandgap ( $E_{\mathrm{g}}=2.1 \mathrm{eV}$ [4]) semiconductor with outstanding chemical and high-temperature stability that makes it a promising material for a wide range of applications [5]. Under ambient conditions, BP crystallizes in cubic sphalerite $(F-43 m)$ structure with boron and phosphorus tetrahedrally coordinated to each other. Another tetrahedral structure of boron phosphide, hexagonal wurtzite $\left(\mathrm{P}_{3} \mathrm{mc}\right)$ polymorph, has also been reported [6], however, in the literature there is no data on the structure and properties of this phase.

The main limitation for the use of boron phosphide is the lack of relatively simple and economical methods of its production, especially, of BP single crystals. The disadvantages of existing methods are the use of toxic and aggressive reagents, complicated technical implementation and high time consumption. Recently, two new methods of boron phosphide synthesis have been developed i.e.

\footnotetext{
*Corresponding author (vladimir.solozhenko@univ-paris13.fr)
} 
self-propagated high-temperature synthesis [7] and mechanochemical synthesis [8] that are characterized by simplicity, high efficiency, low cost and good perspectives for large-scale production. Here we report the results of transmission electron microscopy studies of BP produced by self-propagated high-temperature synthesis.

\section{Experimental}

Microcrystalline powder of boron phosphide has been synthesized by self-propagating hightemperature reaction of boron phosphate and metallic magnesium: $\mathrm{BPO}_{4}+4 \mathrm{Mg}=\mathrm{BP}+4 \mathrm{MgO}$ using the method described earlier [7]. According to X-ray diffraction study (TEXT 3000 Inel, $\mathrm{CuKa1}$ radiation) the sample is well-crystallized sphalerite BP with lattice parameter $a=0.45356(9) \mathrm{nm}$, which was close to the literature value $0.4538(2) \mathrm{nm}$ [9]. The amount of $\mathrm{B}_{12} \mathrm{P}_{2}$ boron subphosphide impurity caused by a partial decomposition of as-forming BP due to a high local temperatures in the reaction mixture did not exceed 1 vol.\%.

Microstructure of boron phosphide has been studied by high-resolution transmission electron microscopy (HRTEM) using JEM-2010 microscope with energy dispersive X-ray spectroscopy (EDS) attachment.

\section{Results and discussion}

According to TEM data (Fig. 1), the boron phosphide powder consists of flat polyhedral particles having dimensions from 50 to several hundred nanometers; and a significant part of them contains twins. Fig. 2 presents TEM and HRTEM images of sphalerite BP particle containing two systems of twins. White rectangles depict stacking faults which change the layer stacking sequence from $A B C A B C$ for cubic (3C) sphalerite structure to $A B A B A B A$ for hexagonal $(2 \mathrm{H})$ wurtzite structure. The corresponding fast Fourier transform (FFT) image is shown in the inset. Similar structural transformations were previously observed in carbon (diamond $\rightarrow$ lonsdaleite) [10] and in silicon $(\mathrm{SiI} \rightarrow \mathrm{SiIV}$ ) [11]. According to [12], the lattice parameters of wurtzite BP are $a=0.320 \mathrm{~nm}$ and $c=0.531 \mathrm{~nm}$, which are noticeably different from the lattice parameters of wurtzite $\mathrm{BN}$ $(a=0.25505 \mathrm{~nm} ; c=0.4210 \mathrm{~nm}[13])$.

Fig. 3 shows a fragment of BP particle containing two phases. The crystal lattice in the lower part corresponds to the sphalerite (3C) structure with lattice parameter $a=0.4538 \mathrm{~nm}$, whereas the structure in the upper part is a rhombohedral (3R) lattice with tripled value of interplanar distance for (111) sphalerite planes i.e. $0.262 \times 3=0.786 \mathrm{~nm}$. The tripling is visible both in HRTEM and FFT images. Stacking faults and polytypes are not observed in this structure. 
As follows from the comparison of FFT images of two neighboring fragments (see insets in Fig. 3), the $(-111)_{3 \mathrm{C}} \|(001)_{3 \mathrm{R}}$ orientation relation is observed. According to our assessment, the lattice parameters of the rhombohedral phase are $a=0.321 \mathrm{~nm}, c=0.786 \mathrm{~nm}$.

We could not find any information on the existence of rhombohedral BP in the literature, however, there is the experimental evidence for formation of rhombohedral polymorphic modification of zinc sulfide $(\mathrm{ZnS})$ [14], the structural analogue of BP. This three-layer rhombohedral (3R) structure of $\mathrm{ZnS}$ was found to occur in the $600-1020^{\circ} \mathrm{C}$ temperature range as an intermediate phase between sphalerite $(3 \mathrm{C})$ and wurtzite $(2 \mathrm{H})$ structures. In our case, the appearance of rhombohedral BP can be associated with deformation of as-formed sphalerite lattice in the course of ultrafast self-propagated high-temperature reaction, which leads to a change of interplanar distances for $\{111\}_{3 \mathrm{C}}$ planes and, consequently, results in the change of the lattice symmetry.

Fig. 4a shows a two-phase particle. The left part of the particle is of $\mathrm{B}_{12} \mathrm{P}_{2}$ composition and contains polytypes, as evidenced by the elongated streaks passing through the spots in the corresponding FFT image (Fig. 4b). The right part contains a twice-twinned fragment of BP composition (the corresponding FFT image is shown in Fig. 4c). The mutual orientation of these two phases corresponds to the $(003)_{\mathrm{B}_{12} \mathrm{P}_{2}} \|(111)_{\mathrm{BP}}$ orientation relation. The lower part of $\mathrm{BP}$ fragment transforms into $\mathrm{B}_{12} \mathrm{P}_{2}$ without a coherent boundary. The arrows denote the $\{111\}_{\text {BP }}$ planes. One of these planes separates $\mathrm{B}_{12} \mathrm{P}_{2}$ and $\mathrm{BP}$ crystals, while the other two are twinning planes in BP crystal. Such a junction of twinning planes is generally denoted as $\Sigma=9$ [15]. In other words, $\Sigma=9$ (second order twin) boundary is created when two $\Sigma=3$ (first order twin) boundaries intersect.

Previously, in boron subphosphide $\mathrm{B}_{12} \mathrm{P}_{2}$ synthesized by the same self-propagating hightemperature method we have discovered two systems of twins i.e. conventional twins on the $(0003)_{h}$ plane and nanotwins resulting from duplication of the rhombohedral unit cell of $\mathrm{B}_{12} \mathrm{P}_{2}$ along one of the basic vectors [16]. In the present study, neither these nor other twins were observed in the only few $\mathrm{B}_{12} \mathrm{P}_{2}$ particles found. The latter can be explained by the fact that $\mathrm{B}_{12} \mathrm{P}_{2}$ is a secondary phase formed due to the thermal decomposition of as-synthesized BP at points of the local overheating in the reaction front.

Fig. 5 shows a BP particle with a complex structure. In the upper part of HRTEM image, twins along the (111) plane are observed. In the lower part on the right, twins along the (11-1) plane are visible. The rhombohedral (3R) phase was formed on both sides of the (111) plane in the lower part of the image. On the right side the tripling occurred along the [11-1 $]_{3 \mathrm{C}}$ direction. On the left part along the $[111]_{3 \mathrm{C}}$ direction. Thus, Fig. 5 shows a twinned particle of sphalerite BP which also contains two fragments of rhombohedral phase. The rhombohedral phase in the right part of the image is formed as a result of twinning. 


\section{Conclusions}

In boron phosphide produced by self-propagated high-temperature synthesis, along with traditional sphalerite $(3 \mathrm{C})$ polymorphic modification, fragments of wurtzite $(2 \mathrm{H})$ polymorph and previously unknown for BP rhombohedral (3R) structure were found which shred new light on polymorphism of boron phosphide.

\section{Acknowledgments}

V.L.S. gratefully acknowledges support from the Agence Nationale de la Recherche (projet $\mathrm{N}^{\circ}$ ANR-20-ASTR-0020).

\section{ORCID IDs}

Vladimir L. Solozhenko (D) https://orcid.org/0000-0002-0881-9761 


\section{References}

[1] V.L. Solozhenko, V. Bushlya, Mechanical properties of boron phosphides. J. Superhard Mater., 41 [2] 84-89 (2019).

[2] V.L. Solozhenko, V.A. Mukhanov, On melting of boron phosphide under pressure. J. Superhard Mater., 37 [6] 438-439 (2015).

[3] V.L. Solozhenko, O.O. Kurakevych, Y. Le Godec, A.V. Kurnosov, A.R. Oganov, Boron phosphide under pressure: in situ study by Raman scattering and X-ray diffraction. J. Appl. Phys., 116 [3] 033501 (2014).

[4] A. Agui, S. Shin, Y. Kumashiro, Electronic structure of BP studied by resonant soft X-ray emission spectroscopy. J. Phys. Soc. Japan, 68 [1] 166-169 (1960).

[5] K. Woo, K. Lee, K. Kovnir, BP: synthesis and properties of boron phosphide. Mater. Res. Express, 3 [7] 074003 (2016).

[6] P. Villars, L.D. Calvert, Pearson's Handbook of Crystallographic Data for Intermetallic Phases, American Society for Metals, Metals Park, OH, 1985.

[7] V.A. Mukhanov, P.S. Sokolov, Y. Le Godec, V.L. Solozhenko, Self-propagating hightemperature synthesis of boron phosphide. J. Superhard Mater. 35 [6] 415-417 (2013).

[8] V.A. Mukhanov, D. Vrel, P.S. Sokolov, Y. Le Godec, V.L. Solozhenko, Ultra-fast mechanochemical synthesis of boron phosphides, $\mathrm{BP}$ and $\mathrm{B}_{12} \mathrm{P}_{2}$. Dalton Trans., 45 [25] 10122-10126 (2016).

[9] S. Rundqvist, Crystal structure of boron phosphide BP. Congres International de Chimie pure et applique, Mem. Sect. 1957, 539 (1958).

[10] B. Kulnitskiy, I. Perezhogin, G. Dubitsky, V. Blank, Polytypes and twins in the diamondlonsdaleite system formed by high-pressure and high-temperature treatment of graphite. Acta Cryst. B, 69, 474-479 (2013).

[11] B. Kulnitskiy, M. Annenkov, I. Perezhogin, M. Popov, D. Ovsyannikov, V. Blank, Mutual transformation between crystalline phases in silicon after treatment in a planetary mill: HRTEM studies. Acta Cryst. B, 72, 733-737 (2016).

[12] H. Momida, T. Oguchi, Effects of lattice parameters on piezoelectric constants in wurtzite materials: A theoretical study using first-principles and statistical-learning methods. Appl. Phys. Express, 11, 041201 (2018).

[13] A.V. Kurdyumov, V.L. Solozhenko, W.B. Zelyavski, Lattice parameters of boron nitride polymorphous modifications as a function of their crystal-structure perfection. J. Appl. Crystallogr., 28, 540-545 (1995).

[14] D.C. Buck, L.W. Strock, Trimorphism in zinc sulfide. Am. Mineral., 40 [3-4] 192-200 (1955). 
[15] J. Narayan, Dislocations, twins, and grain boundaries in CVD diamond thin films: Atomic structure and properties. J. Mater. Res., 5 [11] 2414-2423 (1990).

[16] B.A. Kulnitskiy, I.A. Perezhogin, V.D. Blank, V.A. Mukhanov, V.L. Solozhenko, Nanotwinning in boron subphosphide $\mathrm{B}_{12} \mathrm{P}_{2}$. J. Superhard Mater., 41 [2] 139-141 (2019). 


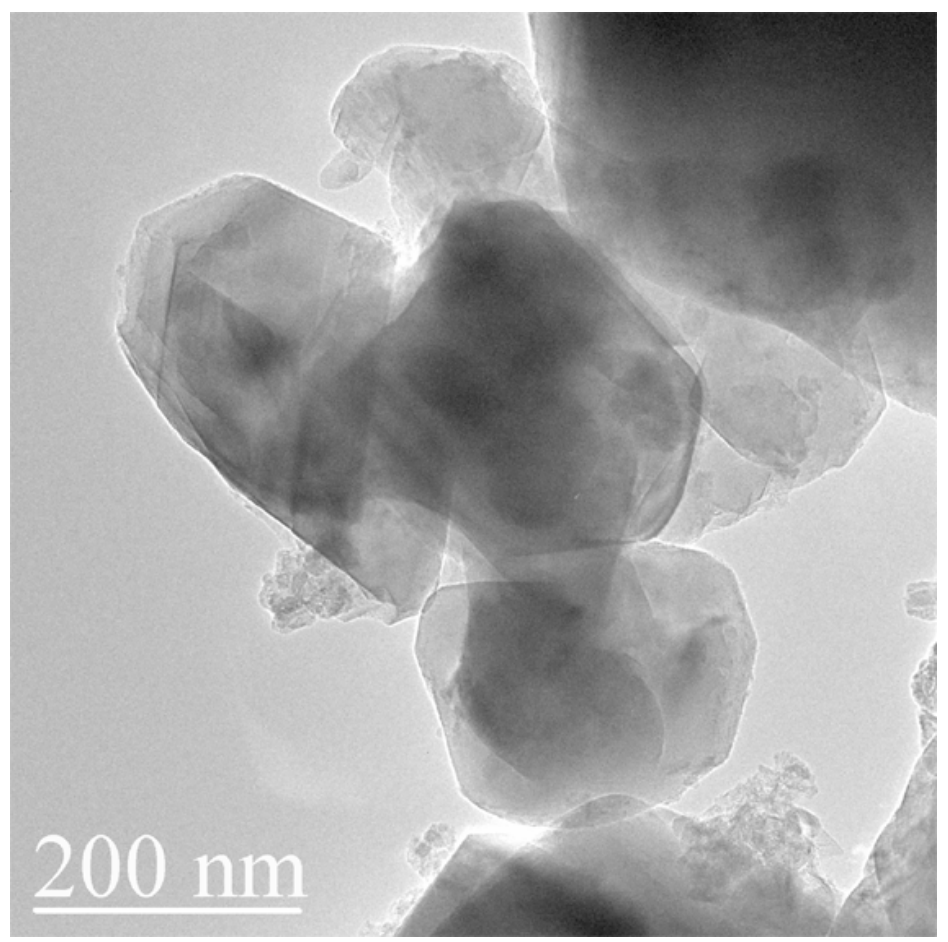

Fig. 1. A characteristic TEM image of boron phosphide particles. 

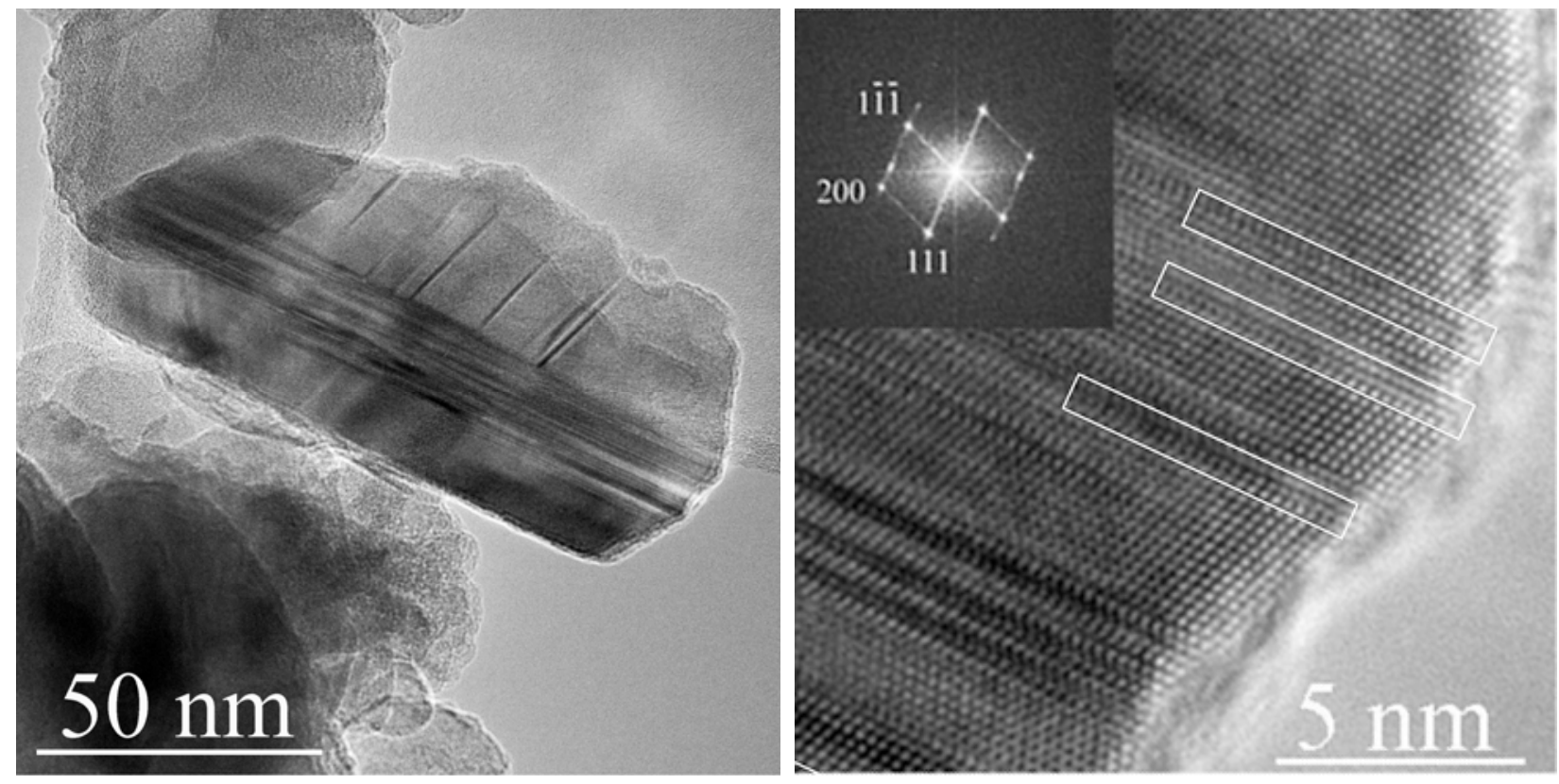

Fig. 2. Two systems of twins in BP structure: $(a)$ overview of the particle and $(b)$ atomic resolution image of a portion of the particle where white rectangles depict the fragments of wurtzite $(2 \mathrm{H})$ structure with $A B A B A B A$ layer stacking sequence; the corresponding FFT image is shown in the inset. 


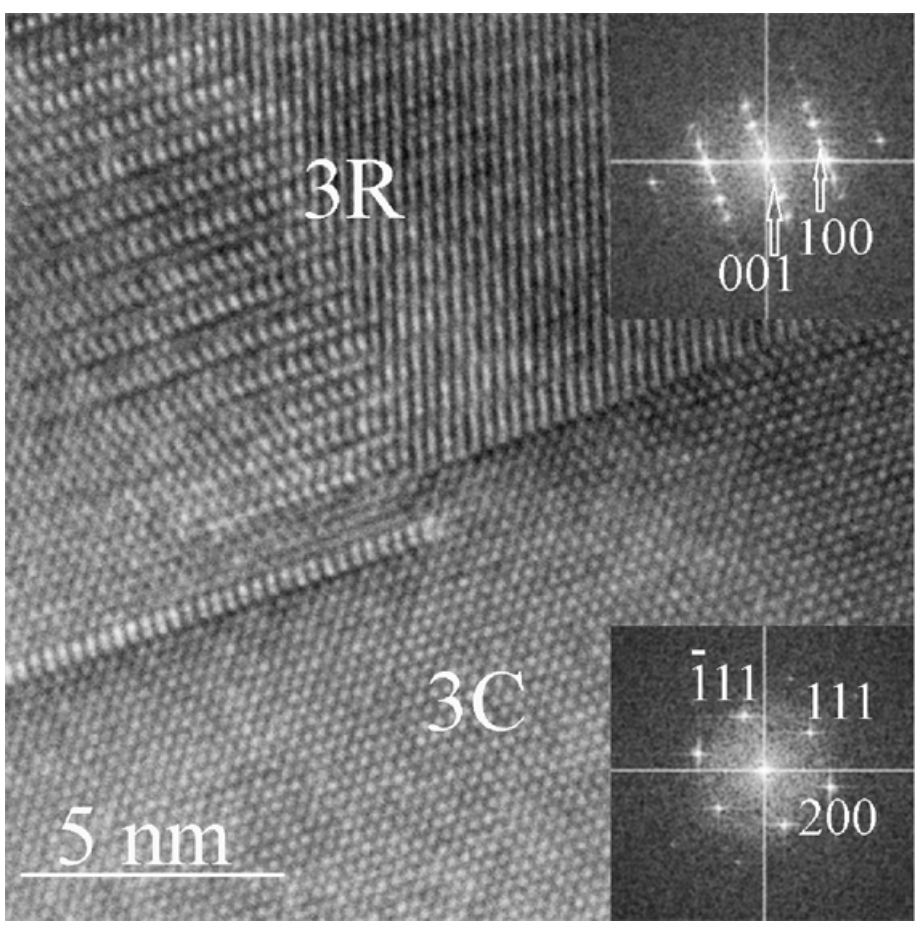

Fig. 3. HRTEM image and corresponding FFT images of the fragment of BP grain containing two phases: rhombohedral (3R) structure (the upper half) and sphalerite (3C) structure (the lower half). 

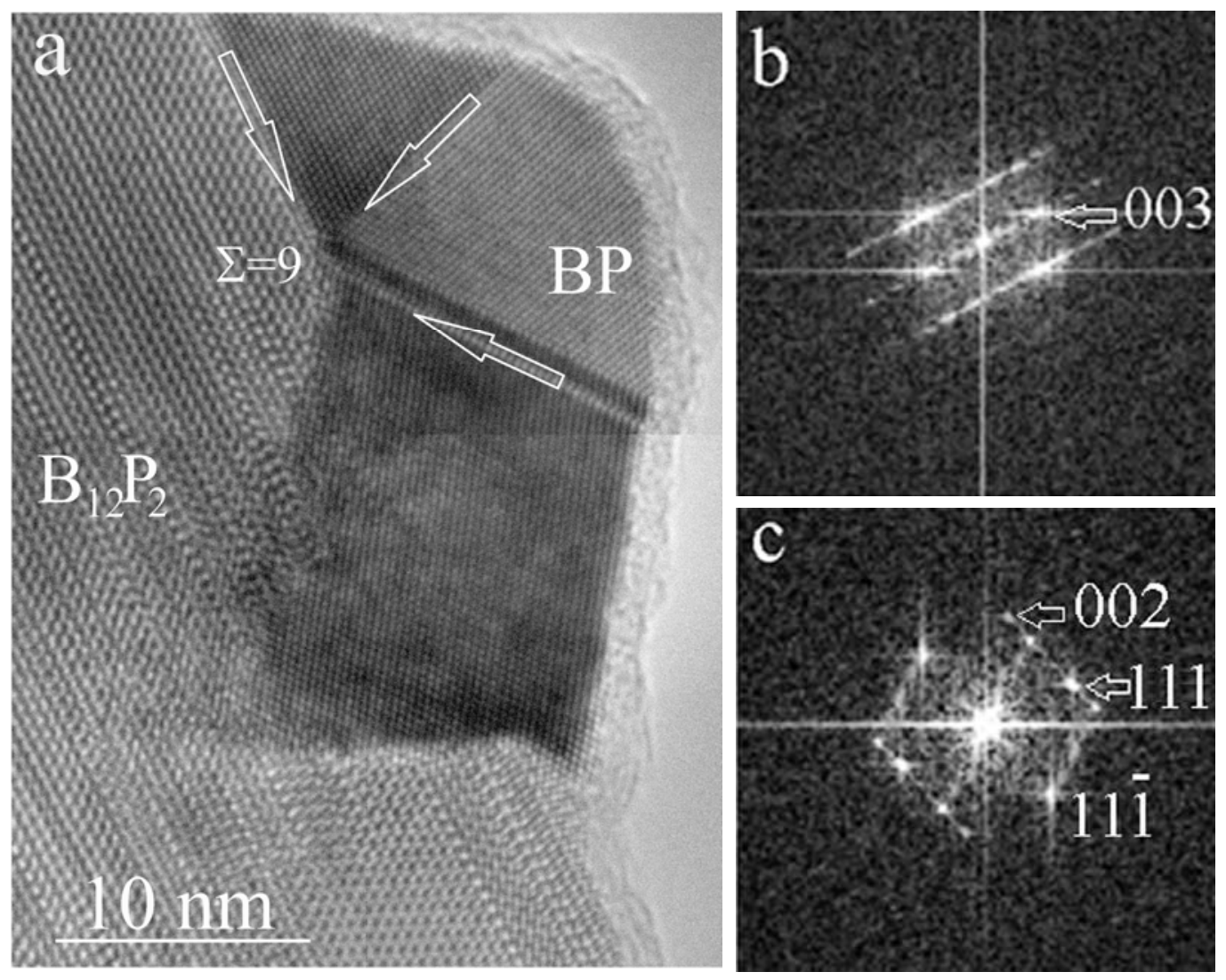

Fig. 4. (a) HRTEM image of two-phase particle: the left part is of $\mathrm{B}_{12} \mathrm{P}_{2}$ composition and contains polytypes; the right part is twice-twinned fragment of BP composition. The arrows indicate $\{111\}_{\text {BP }}$ planes. (b) FFT image of the left part of $(a)$; (c) FFT image of the right part of $(a)$. 

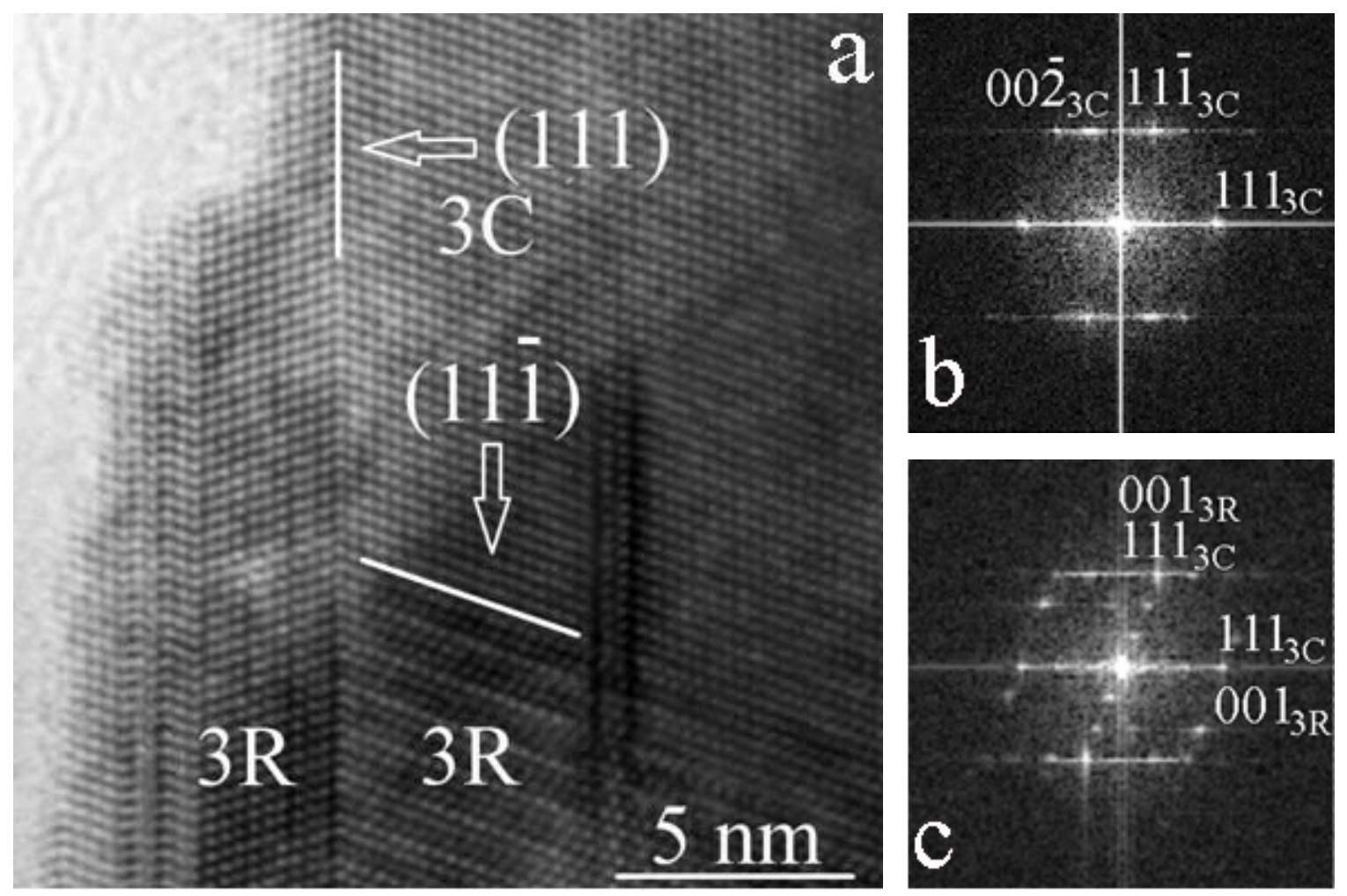

Fig. 5. (a) HRTEM image of sphalerite (3C) BP particle which also contains two fragments of rhombohedral (3R) polymorph: the upper part shows BP twins on the $(111)_{3 \mathrm{C}}$ plane; (b) the corresponding FFT-image. At the lower part on both sides of the $(111)_{3 \mathrm{C}}$ plane (vertical white dash), two fragments of rhombohedral phase were formed. On the right side the tripling occurred relative to the (11-1) 3 C plane; while on the left side the tripling occurred relative to the $(111)_{3 \mathrm{C}}$ plane. (c) FFT image of the right fragment of rhombohedral (3R) structure. 